\title{
FLAIR signal increase of the fluid within the resection cavity after glioma surgery: generally valid as early recurrence marker?
}

\author{
Stefanie Bette, MD, ${ }^{1}$ Jens Gempt, MD, ${ }^{2}$ Thomas Huber, MD, ${ }^{1}$ Claire Delbridge, MD, ${ }^{3}$ \\ Bernhard Meyer, MD, ${ }^{2}$ Claus Zimmer, MD, Jan S. Kirschke, MD, ${ }^{1}$ and Tobias Boeckh-Behrens, MD1 \\ Departments of ${ }^{1}$ Neuroradiology, ${ }^{2}$ Neurosurgery, and ${ }^{3}$ Neuropathology, Klinikum rechts der Isar, Technische Universität \\ München, Germany
}

\begin{abstract}
OBJECTIVE Recent studies have indicated that a signal intensity increase of the fluid within the resection cavity on FLAIR images may predict tumor recurrence after glioma surgery. The aim of this study was to assess the increase in FLAIR signal intensity in a large patient cohort and in subgroups to assess its prognostic value for early tumor recurrence in glioma patients.

METHODS A total of 212 patients (213 cases) who had undergone surgery for an intracranial glioma (WHO Grade IV [ $n=103]$, WHO Grade III [ $n=57]$, and WHO Grade II [ $n=53]$ ) were included in this retrospective study. FLAIR signal within the resection cavity at the time of tumor recurrence/last contact and on the previous MRI study was assessed qualitatively and quantitatively. Appearance of FLAIR signal increase was studied over time using Kaplan-Meier estimates in subgroups.
\end{abstract}

RESULTS Patients with WHO Grade II glioma and connection of the resection cavity to CSF who did not undergo radiotherapy did not regularly develop this sign and were excluded from further analysis. For the remaining 87 cases, FLAIR signal intensity increase was observed in 27 cases. Recurrent disease was found in 26 of these 27 cases, resulting in a specificity of $80.0 \%$, a sensitivity of $31.7 \%$, and positive and negative predictive values of $96.3 \%$ and $6.7 \%$, respectively. In 4 cases this sign had been observed prior (range 2.8-8.5 months) to tumor recurrence defined by standard criteria. Quantitative analysis underlined the results of qualitative analysis, but it did not add a diagnostic value.

CONCLUSIONS Signal intensity increase of the fluid within the resection cavity on FLAIR images is a rare but highly specific and early sign for tumor recurrence/tumor progression in completely and incompletely resected high-grade glioma without connection of the resection cavity to CSF and with radiotherapy.

https://thejns.org/doi/abs/10.3171/2016.8.JNS16752

KEY WORDS glioma; signal increase; tumor recurrence; FLAIR; resection cavity; oncology

I NTRACRANIAL gliomas are infiltrating tumors with a high recurrence rate, despite advances in diagnosis and treatment. 1,14,24,30,32,34,35 In particular, patients with highgrade gliomas - Grades III and IV according to the 2007 WHO classification ${ }^{16}$ - show a poor prognosis with limited overall survival. ${ }^{30,32,34,35}$ Complete neurosurgical tumor resection with preservation of neurological function has been shown to be an important prognostic factor for lowgrade and high-grade gliomas..$^{3,25,26,28}$ As gliomas are infiltrating tumors with indistinct borders, many tumors can only be partially resected. ${ }^{19,22}$ MRI is important for moni- toring therapy effects and detection of tumor recurrence/ progression after surgery. Tumor progression on MRI studies is defined according to the response assessment in neurooncology (RANO) criteria. ${ }^{31,33}$ Early detection of tumor recurrence/tumor progression in glioma patients is important to adapt surveillance intervals and to adjust therapy regimens in a timely manner. Recent studies have shown that signal increase of the fluid within the resection cavity on T2-weighted FLAIR sequences predicts early tumor recurrence in partially resected glioma with a high specificity. ${ }^{11,36}$ However, not only were the patient cohorts

ABBREVIATIONS FET = 0-(2-[18F]fluoroethyl)-L-tyrosine; IQR = interquartile range; MPRage = magnetization prepared rapid gradient echo; NPV = negative predictive value; PPV = positive predictive value; RANO = response assessment in neurooncology; $\mathrm{ROI}=$ region of interest.

SUBMITTED March 23, 2016. ACCEPTED August 5, 2016.

INCLUDE WHEN CITING Published online October 21, 2016; DOI: 10.3171/2016.8.JNS16752. 
relatively small in these studies, no analysis of subgroups regarding patients with or without previous radiotherapy, with complete and incomplete tumor resection, and different WHO grades was performed. ${ }^{11,36}$

The aim of this study was to assess the prognostic value of signal increase on FLAIR sequences of the fluid within the resection cavity in a large patient cohort for tumor recurrence in intracranial glioma and to address the abovementioned subgroups.

\section{Methods \\ Patient Population}

This retrospective noninterventional single-center study was approved by the local ethics committee in accordance with the ethical standards of the 1964 Declaration of Helsinki and its later amendments. ${ }^{9}$

A total of 299 consecutive patients who had undergone 300 surgeries for an intracranial glioma (recurrent or primary disease) between January 2008 and October 2015 were analyzed. All patients included in this analysis had undergone early postoperative MRI $(<72$ hours after surgery). To avoid bias due to blood and postoperative fluid, analysis was not performed on early postoperative MRI ( $<72$ hours after surgery), and only patients with at least 2 MR images obtained after the early postoperative MRI study were included.

Patients with missing follow-up information $(n=35)$, diameter of the resection cavity smaller than the intended region of interest (ROI) size due to shrinking of the resection cavity after surgery $(\mathrm{n}=39)$, infection in the resection cavity $(\mathrm{n}=2)$, missing FLAIR sequence $(\mathrm{n}=1)$, current bevacizumab therapy $(\mathrm{n}=1)$, or solid recurrent tumor filling the entire resection cavity $(\mathrm{n}=1)$ were excluded. Patients who were lost to follow-up and did not reach the study end point (recurrent disease or death, $\mathrm{n}=8$ ) were also excluded. Therefore, 212 patients who had undergone 213 surgeries were analyzed. In 1 patient, 2 different resection cavities (right and left hemispheres) were analyzed and assessed as 2 different cases. Surgery was performed with the aim of maximum tumor resection while preserving eloquent areas such as language and motor function. Extent of resection was assessed on the early postoperative MRI; complete resection was defined as total resection of the contrast-enhancing tumor part in high-grade glioma or total resection of the FLAIR hyperintense tumor in low-grade glioma. Histopathological evaluation was done based on the 2007 WHO criteria for CNS tumors. ${ }^{16}$ Date of surgery, date of MRI at recurrent disease/last contact, and date of death as well as date of the previous MRI and date of FLAIR signal intensity change were recorded. Radiotherapy was assessed during the observation period. We defined tumor progression/tumor recurrence as well as death as study end points. Tumor progression was defined according to the RANO criteria in an interdisciplinary consensus (neuroradiology, neurosurgery, neuroradiology, and nuclear medicine). ${ }^{31,33}$ The date of recurrent disease was defined as the date of change in further treatment (e.g., radiotherapy, surgery, or chemotherapy). In unclear cases, advanced imaging methods (including $O-\left(2-\left[{ }^{18} \mathrm{~F}\right]\right.$ fluoroethyl)-L-tyrosine-PET [FET-PET]) were used for diagnosis of tumor progression or tumor recurrence. ${ }^{5} \mathrm{~Pa}-$ tients who died during the observation period without imaging showing recurrent disease were classified as having tumor recurrence.

\section{Magnetic Resonance Imaging}

MRI was performed using Achieva $(\mathrm{n}=111)$ or Ingenia $(\mathrm{n}=8)$ (Philips Medical Systems); Verio $(\mathrm{n}=77)$, Avanto $(n=5)$, Espree $(n=3)$, or Aera $(n=5)$ (Siemens Healthcare); or other MR scanners $(n=4)$. For MRI performed using Achieva MR scanners, axial T2-weighted FLAIR images (acquisition time 3 minutes, TR 12,000 msec, TE $140 \mathrm{msec}, 0.45 \times 0.45 \times 4 \mathrm{~mm}$ spatial resolution) or T2-weighted 3D FLAIR images (acquisition time 4 minutes and 52 seconds, TR $4800 \mathrm{msec}$, TE $278 \mathrm{msec}, 1.04$ $\times 1.04 \times 1.12 \mathrm{~mm}$ spatial resolution) as well as pre- and postcontrast axial T1-weighted fast field echo images (acquisition time 2 minutes and 53 seconds, TR $530 \mathrm{msec}$, TE $10 \mathrm{msec}, 0.45 \times 0.45 \times 4 \mathrm{~mm}$ spatial resolution) or preand postgadolinium magnetization prepared rapid gradient echo (MPRage) images (acquisition time 5 minutes and 55 seconds, TR $9 \mathrm{msec}$, TE $4 \mathrm{msec}, 1 \mathrm{~mm}^{3}$ isotropic spatial resolution) were acquired. For sequences obtained using the Verio MR scanner, axial T2-weighted FLAIR images (acquisition time 3 minutes and 44 seconds, TR $8560 \mathrm{msec}$, TE $136 \mathrm{msec}, 0.8 \times 0.7 \times 4 \mathrm{~mm}$ spatial resolution) or 3D FLAIR (acquisition time 5 minutes and 52 seconds, TR $5000 \mathrm{msec}$, TE $395 \mathrm{msec}, 1 \mathrm{~mm}^{3}$ isotropic spatial resolution) and axial T1-weighted images pre- and postgadolinium (T1 inversion recovery, time of inversion $860 \mathrm{msec}$, acquisition time 4 minutes and 2 seconds, TR $2000 \mathrm{msec}$, TE $9 \mathrm{msec}, 0.9 \times 0.7 \times 4 \mathrm{~mm}$ spatial resolution) or MPRage (acquisition time 4 minutes and 18 seconds, TR $1900 \mathrm{msec}$, TE $2.45 \mathrm{msec}, 1.1 \times 1.1 \times 1 \mathrm{~mm}$ spatial resolution) were assessed. The contrast agent (Magnograf or Magnevist) was administered intravenously $(0.2 \mathrm{ml} / \mathrm{kg}$, $0.5-1 \mathrm{ml} / \mathrm{sec}$ ), using an MR-compatible contrast medium injection system (Spectris Solaris EP, Siemens Medical). Axial and coronal T2-weighted FLAIR images were acquired in 117 cases and 3D FLAIR images in 96 cases.

\section{Image Analysis}

MR image evaluation was done by a neuroradiologist with 6 years of experience (S.B.). Signal intensity of the fluid within the resection cavity on FLAIR images was assessed at the date of recurrent disease/last MRI and was classified as having a normal or elevated signal intensity compared with CSF. Change in signal intensity (classified as no change, increase, or decrease) was determined based on comparisons with previous MRI studies. An open connection versus no connection of the resection cavity to CSF was assessed. The date of signal intensity change was recorded, and the times from surgery to signal intensity change and from change to recurrent disease were calculated.

Quantitative analysis was performed in a manner similar to a previously described ROI method ${ }^{36}$ ROIs with a size of 5-10 $\mathrm{mm}$ were drawn 1) in the resection cavity, 2 ) in the CSF, and 3) in extracranial space to avoid background noise (Fig. 1). Only fluids and no solid components 


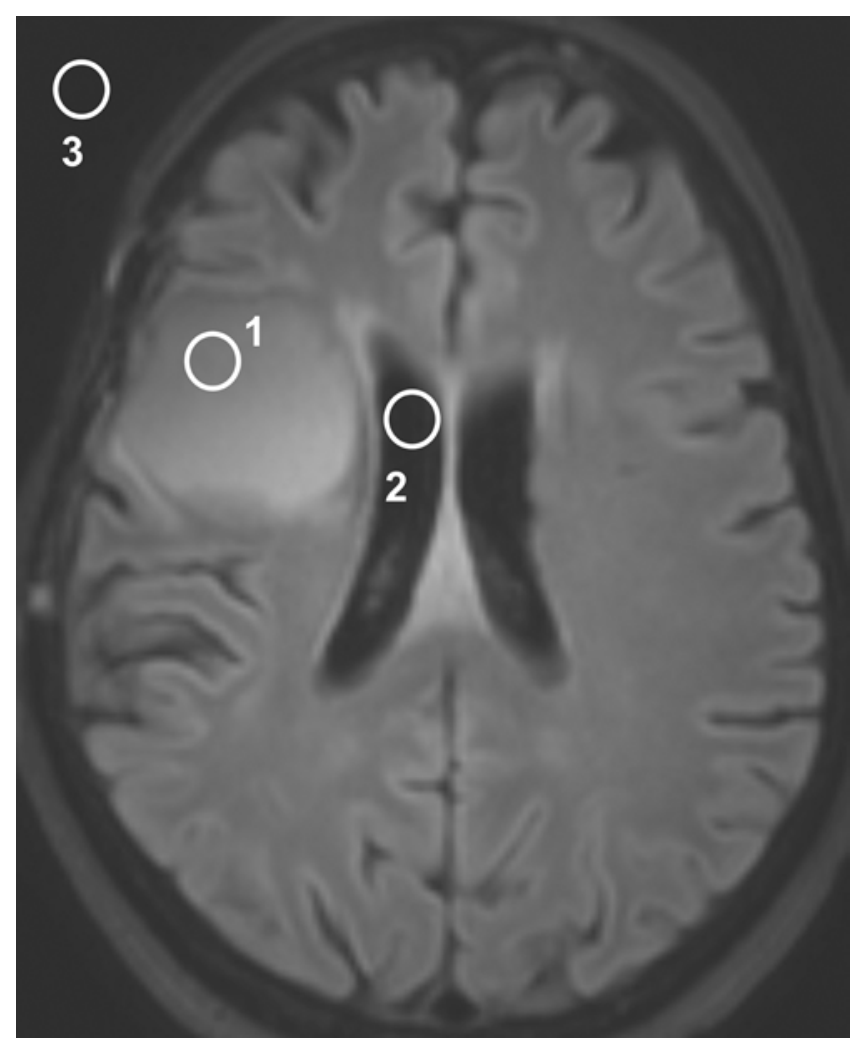

FIG. 1. Quantitative analysis of signal intensity using ROls in the resection cavity (1), CSF (2), and extracranial space (3).

in the resection cavity were measured. Ratios of ROIs $1 / 2$ and $1 / 3$ were calculated. ROIs were also measured on the MR image before signal intensity change or on the previous MR image in cases with no change. Differences in signal intensity were calculated for ROIs measured in the resection cavity (1) and for ratios $1 / 2$ and $1 / 3$ and then again were classified as no change/increase or decrease. To assess interrater reliability a second reader (T.H., 3 years of experience) performed qualitative and quantitative analyses, Cohen's kappa was calculated for agreement of qualitative analysis, and the intraclass correlation coefficient was calculated for agreement of quantitative analysis. In cases of discrepancy, a third reader (T.B., 12 years of experience) decided.

\section{Statistical Analysis}

Statistical analysis including descriptive data analysis was performed using IBM SPSS Statistics (version 23.0, IBM Corp.). Nonnormally distributed data are shown as the median (interquartile range [IQR]). The Mann-Whitney U-test was performed between the 2 groups (recurrent or no recurrent disease). Occurrence of FLAIR signal increase over time and the time from FLAIR signal increase to tumor recurrence were studied over time using KaplanMeier estimates.

Specificity, sensitivity, positive predictive value (PPV), and negative predictive value (NPV) were calculated for FLAIR signal increase and tumor recurrence/tumor recurrence on the next MRI after a FLAIR signal change was noted. A $p$ value $<0.05$ was defined as significant.
TABLE 1. Baseline patient and tumor characteristics

\begin{tabular}{lc}
\hline \multicolumn{1}{c}{ Variable } & Value \\
\hline Mean age in yrs & $49.2 \pm 14.9$ \\
\hline Female sex & $88 / 213(41.3)$ \\
\hline Histopathology & \\
Glioblastoma (WHO IV) & $103 / 213(48.4)$ \\
Anaplastic glioma (WHO III) & $57 / 213(26.8)$ \\
Astrocytoma & $36 / 57(63.2)$ \\
Oligoastrocytoma & $18 / 57(31.6)$ \\
Oligodendroglioma & $3 / 57(5.3)$ \\
Low-grade glioma (WHO II) & $53 / 213(24.9)$ \\
Astrocytoma & $37 / 53(69.8)$ \\
Oligoastrocytoma & $9 / 53(17.0)$ \\
Oligodendroglioma & $6 / 53(11.3)$ \\
PXA & $1 / 53(1.9)$ \\
\hline Recurrent disease during FU & $169 / 213(79.3)$ \\
Connection resection cavity to CSF & $93 / 213(43.7)$ \\
Radiotherapy & $150 / 213(70.4)$ \\
\hline
\end{tabular}

$\mathrm{FU}=$ follow-up; $\mathrm{PXA}=$ pleomorphic xanthoastrocytoma.

Values are presented as the number of patients (\%) unless indicated otherwise. The mean value represents the mean \pm SD.

\section{Results}

\section{Patient Population and Tumor Characteristics}

The study population consisted of 213 cases (125 males and 88 females, mean age at time of surgery $49.2 \pm 14.9$ years; initial diagnosis: $\mathrm{n}=150$ ) with the diagnosis of an intracranial glioma and previous surgery (Table 1). Of these 213 cases, 132 exhibited complete tumor resection (complete resection of the contrast-enhancing tumor part in high-grade gliomas and complete resection of the FLAIRhyperintense tumor part in low-grade gliomas), and 81 showed incomplete resection. Histopathological analysis revealed the diagnosis of 103 glioblastomas (WHO Grade IV), 57 anaplastic gliomas (WHO Grade III), and 53 lowgrade gliomas (WHO Grade II). Radiotherapy was used in 150 of the 213 cases during the observation period.

A total of 169 of 213 cases presented with recurrent disease during follow-up; patients in 96 cases died during the observation period. The median observation period was 22.0 months for all patients, 15.9 months for glioblastoma patients (WHO Grade IV), 26.8 months for patients with anaplastic gliomas (WHO Grade III), and 36.4 months for low-grade glioma patients (WHO Grade II). Recurrent disease was proven histopathologically in 107 of 169 cases, by MRI according to the RANO criteria in 35 cases, or by additional advanced imaging methods (including FET-PET) in 23 cases, and in 4 cases by death during the observation period without imaging of recurrent disease.

\section{Qualitative Analysis}

Seventy-three (34.3\%) cases showed high FLAIR signal (defined as higher FLAIR signal in the resection cavity than CSF) within the resection cavity, whereas 46 of 213 cases $(21.6 \%)$ showed a change in signal intensity. Increase in FLAIR signal intensity was shown in 32 of 213 $(15.0 \%)$ cases, and decrease was seen in $14(6.6 \%)$ cases. 
TABLE 2. Qualitative assessment of signal intensity of the fluid within the resection cavity

\begin{tabular}{lcc}
\hline \multicolumn{1}{c}{ Variable } & $\begin{array}{c}\text { All Cases } \\
(\mathrm{n}=213)\end{array}$ & $\begin{array}{c}\text { Subgroup Analysis } \\
(\mathrm{n}=87)^{*}\end{array}$ \\
\hline FLAIR hyperintensity & $73 / 213(34.3 \%)$ & $63 / 87(72.4 \%)$ \\
Change in signal intensity & $46 / 213(21.6 \%)$ & $39 / 87(44.8 \%)$ \\
$\quad$ Increase & $32 / 213(15.0 \%)$ & $27 / 87(31.0 \%)$ \\
$\quad$ Decrease & $14 / 213(6.6 \%)$ & $12 / 87(13.8 \%)$ \\
\hline Recurrent disease & $169 / 213(79.3 \%)$ & $82 / 85(96.5 \%)$ \\
Increase/recurrent disease & $31 / 169(18.3 \%)$ & $26 / 82(31.7 \%)$ \\
Increase/no recurrent disease & $1 / 44(2.3 \%)$ & $1 / 5(20.0 \%)$ \\
Specificity of signal increase & $97.7 \%$ & $80.0 \%$ \\
Sensitivity of signal increase & $18.3 \%$ & $31.7 \%$ \\
PPV & $96.9 \%$ & $96.3 \%$ \\
NPV & $23.8 \%$ & $6.7 \%$ \\
\hline
\end{tabular}

* Subgroup analysis of patients with WHO Grade III and IV gliomas, no connection of the resection cavity to CSF, and radiotherapy during the observation period.
For analysis of all patients, FLAIR signal increase in the fluid within the resection cavity showed a sensitivity and specificity of $18.3 \%$ and $97.7 \%$, respectively, and a PPV and NPV of $96.9 \%$ and $23.8 \%$, respectively (Table 2). Interrater reliability revealed high concordance between the 2 readers (Cohen's kappa: 94.1\%; asymptomatic standardized error $=0.41$ ).

First, we studied the occurrence of FLAIR signal intensity increase over time in all patients, patients with different WHO grades, with previous complete or incomplete resection, with or without radiotherapy, and with or without connection of the resection cavity to CSF (Fig. 2). Significant differences were observed between different WHO grades $(\mathrm{p}<0.001)$; patients with higher WHO grades developed this sign significantly earlier and more often. We also observed significant differences for patients with or without connection of the resection cavity to CSF $(\mathrm{p}<0.001)$ and for patients with or without irradiation

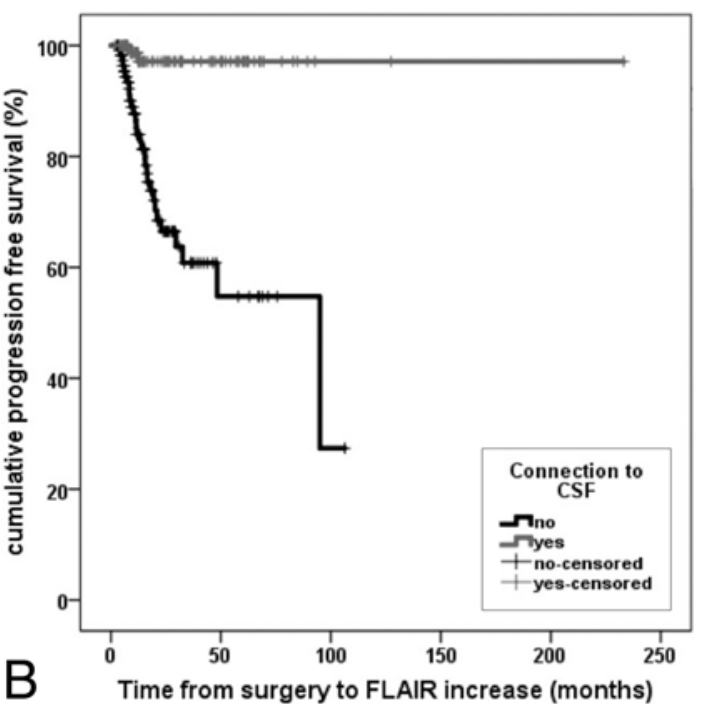

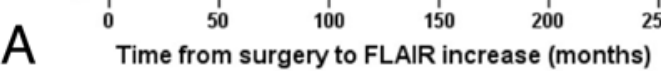

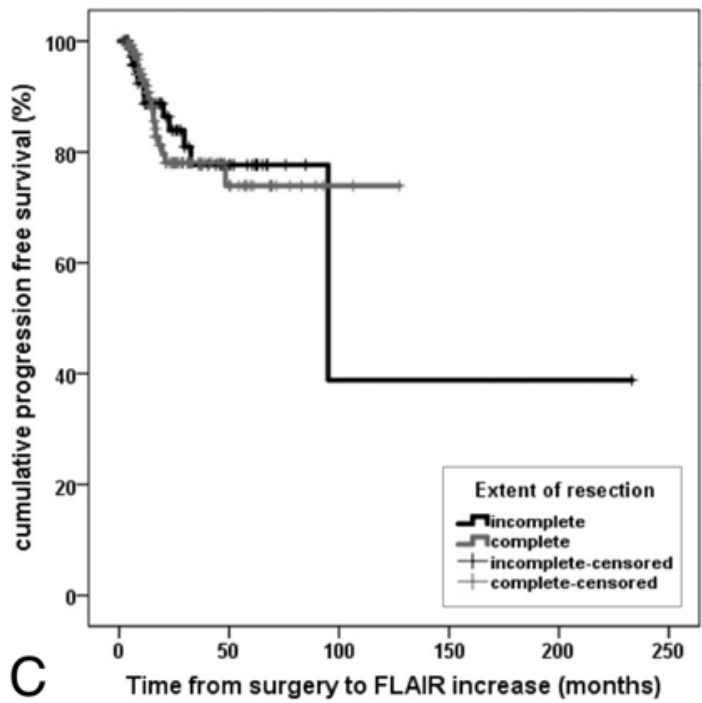

FIG. 2. Kaplan-Meier graphs for time of surgery to FLAIR signal intensity increase within the resection cavity for patients with different WHO grades (A), with/without connection of the resection cavity to CSF (B), complete/incomplete tumor resection (C), and with/without radiotherapy (D). 

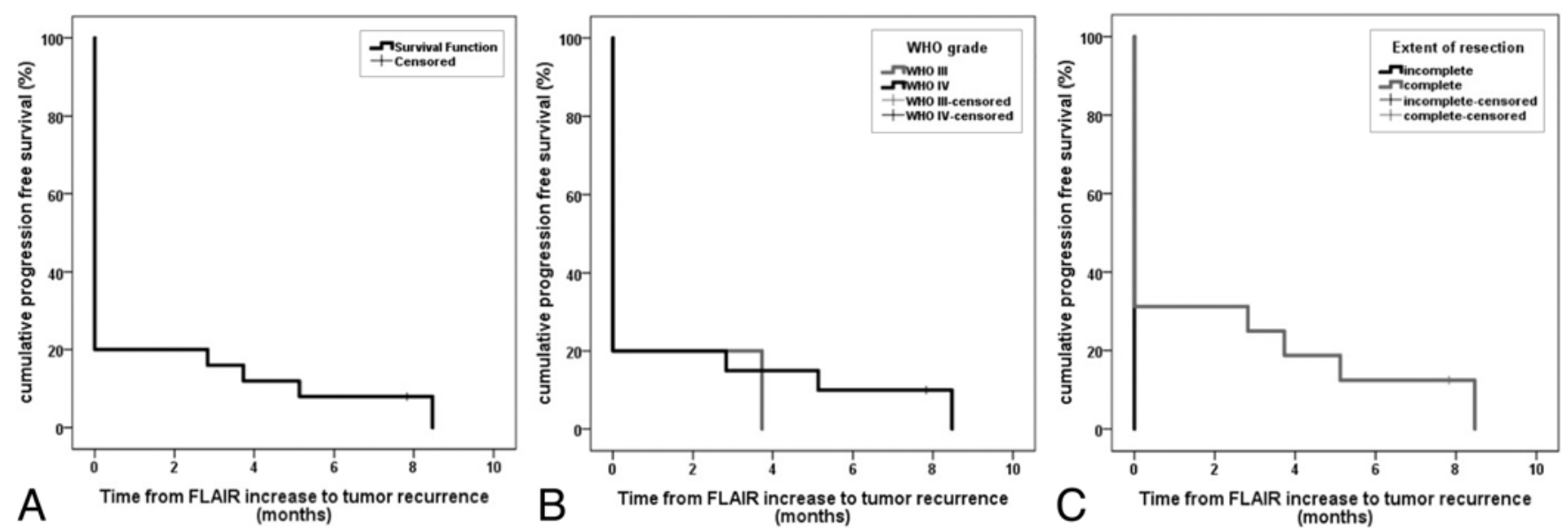

FIG. 3. Kaplan-Meier graphs for time of FLAIR signal intensity increase within the resection cavity to tumor recurrence/progression by standard criteria in all remaining patients $(n=87 ; A)$, different WHO grades $(B)$, and complete/incomplete tumor resection $(C)$.

during the observation period $(\mathrm{p}<0.001)$. We therefore ended up excluding WHO Grade II patients $(\mathrm{n}=53)$, patients with connection of the resection cavity to CSF ( $\mathrm{n}=$ 93), and patients who did not receive radiotherapy (WHO Grade II $=41$, WHO Grade III $=18$, WHO Grade IV $=4$ [63 total]) from further analysis, as these patients do not typically develop this sign. No significant differences were observed between patients with previous complete or incomplete tumor resection $(\mathrm{p}=0.869)$.

A second analysis for the remaining 87 patients (WHO Grade III or IV, no connection of the resection cavity to $\mathrm{CSF}$, and radiotherapy during the observation period) was performed for FLAIR signal increase as the starting point and tumor recurrence by standard criteria as the end point (Fig. 3). This analysis shows that signal increase develops in most patients at the same time as tumor recurrence by standard criteria. Only 5 patients developed this sign earlier (range 2.8-8.5 months) (Fig. 4); 4 of these patients exhibited recurrent disease by standard criteria at the next MRI session and 1 patient developed no tumor recurrence at the next MRI session. According to our criteria, this latter patient was therefore classified as having no tumor recurrence and was censored in the Kaplan-Meier graph. In this analysis, no significant differences were observed between different WHO grades $(p=0.585)$ and complete/ incomplete tumor resection $(\mathrm{p}=0.066)$.

Eighty-two of the 87 remaining cases $(94.3 \%)$ presented with recurrent disease. Twenty-six of these 82 cases (31.7\%) with recurrent disease showed FLAIR signal increase (Fig. 5). FLAIR signal increase was only observed in 1 patient without recurrent disease, resulting in a specificity of $80.0 \%$ and a sensitivity of $31.7 \%$ and a PPV and NPV of $96.3 \%$ and $6.7 \%$, respectively, of FLAIR signal increase.

\section{Quantitative Analysis}

The intraclass correlation coefficient revealed an excellent agreement of both readers (0.974). Quantitative analysis was performed for the remaining 87 cases. Quantitative assessment of signal intensity of the fluid within the resection cavity showed higher values in cases with recur- rent disease than in cases without recurrent disease (median 368.9 [IQR 109.3-744.5] vs 80.0 [IQR 43.0-142.0]) but missed statistical significance $(\mathrm{p}=0.069)$ (Table 3 and Fig. 6). Similar results are shown for the ratios ROI $1 / 2$ and ROI 1/3 and are presented in Supplemental Table 1.

Quantitative assessment of FLAIR signal increase underlined the results of the qualitative analysis but did not improve its diagnostic accuracy (sensitivity $62.2 \%$, specificity $20.0 \%$, PPV 92.7\%, and NPV 3.2\%).

\section{Discussion}

Signal intensity increase of the fluid within the resection cavity on FLAIR sequences can help to predict early tumor recurrence and tumor progression in patients with partially and completely resected gliomas with a high specificity and a high PPV. This sign occurred mainly in high-grade gliomas without connection of the resection cavity to CSF that had undergone radiotherapy and should therefore be applied in this glioma subgroup. Observation of this specific sign may implicate shortening of surveillance intervals in glioma patients to detect tumor progression or tumor recurrence in a timely manner.

A previous study showed that FLAIR signal increase of the fluid within the resection cavity is a highly specific and also early sign for tumor recurrence in partially resected and irradiated gliomas. ${ }^{36}$ However, this study did not include patients with completely resected glioma or without previous radiotherapy and did not differ between WHO grades. The aim of this study was to validate these findings of FLAIR signal increase in a larger patient cohort and to further include patients with nonirradiated and completely resected gliomas WHO Grades II-IV.

In this study we also observed a high specificity of FLAIR signal increase for tumor recurrence/tumor progression, in some cases $(n=4)$ with a range of 2.8-8.5 months before tumor diagnosis by standard criteria. Most cases showed FLAIR signal increase at the time of diagnosis of tumor recurrence/tumor progression by standard criteria. This raises the question of the added value of this sign in these cases. In some cases it might support the di- 

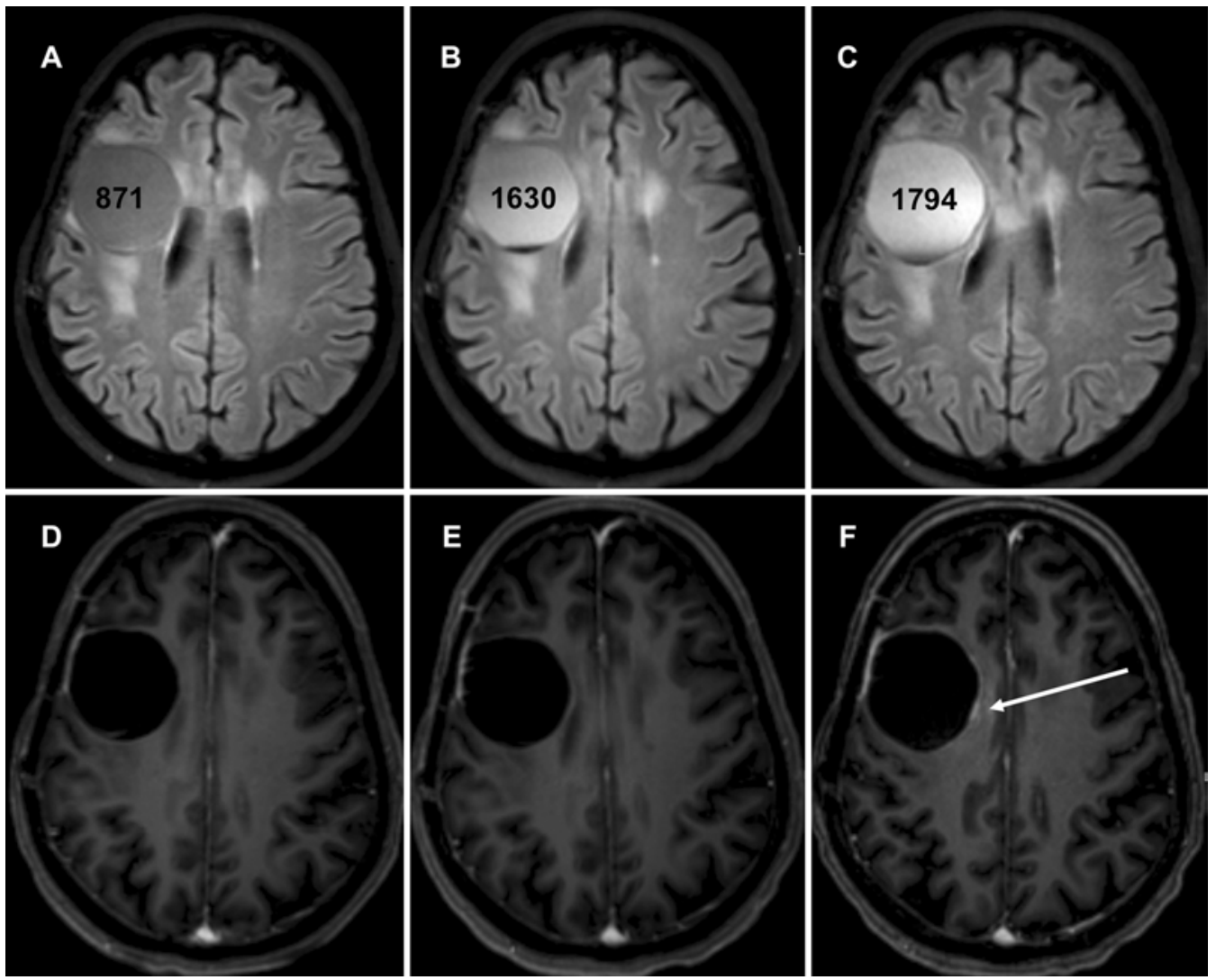

FIG. 4. Case of a patient with the diagnosis of glioblastoma (WHO Grade IV) who had undergone previous surgery and combined radiotherapy. The upper row shows T2-weighted FLAIR images obtained during the follow-up period, and the lower row shows the corresponding T1-weighted postcontrast images. A and D: Images obtained 16 months after surgery. B and E: Images obtained 3 months later, showing an increase of signal intensity on the FLAIR image (B) and no contrast enhancement at this date (E). C and F: Three months later, progression of contrast enhancement was shown (arrow), and diagnosis of recurrent disease was confirmed.

agnosis of tumor recurrence if there is a lack of clarity between progression and pseudoprogression. Even if the sign is only observed rarely prior to standard recurrence criteria, in these cases it might add a diagnostic value, especially if it is included in the routine imaging protocol.

Winterstein et al. postulated that signal intensity increase occurs due to an encapsulation of the resection cavity by tumor cells, leading to a disturbance of CSF exchange and therefore a higher protein concentration. ${ }^{36}$ This hypothesis is supported by the finding in our study that signal intensity increase occurs almost exclusively in cases without connection of the resection cavity to the CSF. Therefore, we hypothesize that in cases with an open connection to the CSF, mixture of CSF and fluid of the resection cavity occurs, leading to a washout of the high protein concentration. These findings also suggest that the sign of signal intensity increase should only be used in cases without connection of the resection cavity to the CSF to increase sensitivity.

Subgroup analysis of gliomas with different WHO grades showed that FLAIR signal increase is observed mainly in high-grade glioma (WHO Grades III and IV).

This finding supports the hypothesis that the failure of the CSF exchange caused by tumor cells is the possible pathomechanism. We hypothesize that tumor cell prolif- eration is higher in WHO Grade IV tumors, leading to a higher tumor cell concentration and therefore a higher disturbance of CSF exchange. Studies with analysis of the composition of the fluid in the resection cavity will have to be performed to understand the pathophysiological mechanisms of the increase in signal intensity.

In the previous study all patients received prior radiotherapy, and it was discussed whether radiation could influence the signal intensity. ${ }^{36}$ Thus, in this study we analyzed cases with and without previous radiotherapy separately. We observed that FLAIR signal increase is mainly observed in patients who received radiotherapy during the observation period. However, this effect might be due to the high number of low-grade gliomas without radiotherapy $(n=41)$ that do not regularly develop this sign and might therefore introduce a bias. Further studies including only high-grade gliomas with or without radiotherapy should be performed to answer this scientific issue.

In this study we included partially and completely resected gliomas and showed that this method could also be used for gliomas that have previously been completely resected. Gliomas are infiltrating tumors with indistinct borders, and tumor margins are hard to define. ${ }^{17}$ The aim of neurosurgical resection is resection of the contrast-enhancing tumor part in high-grade gliomas with preservation 

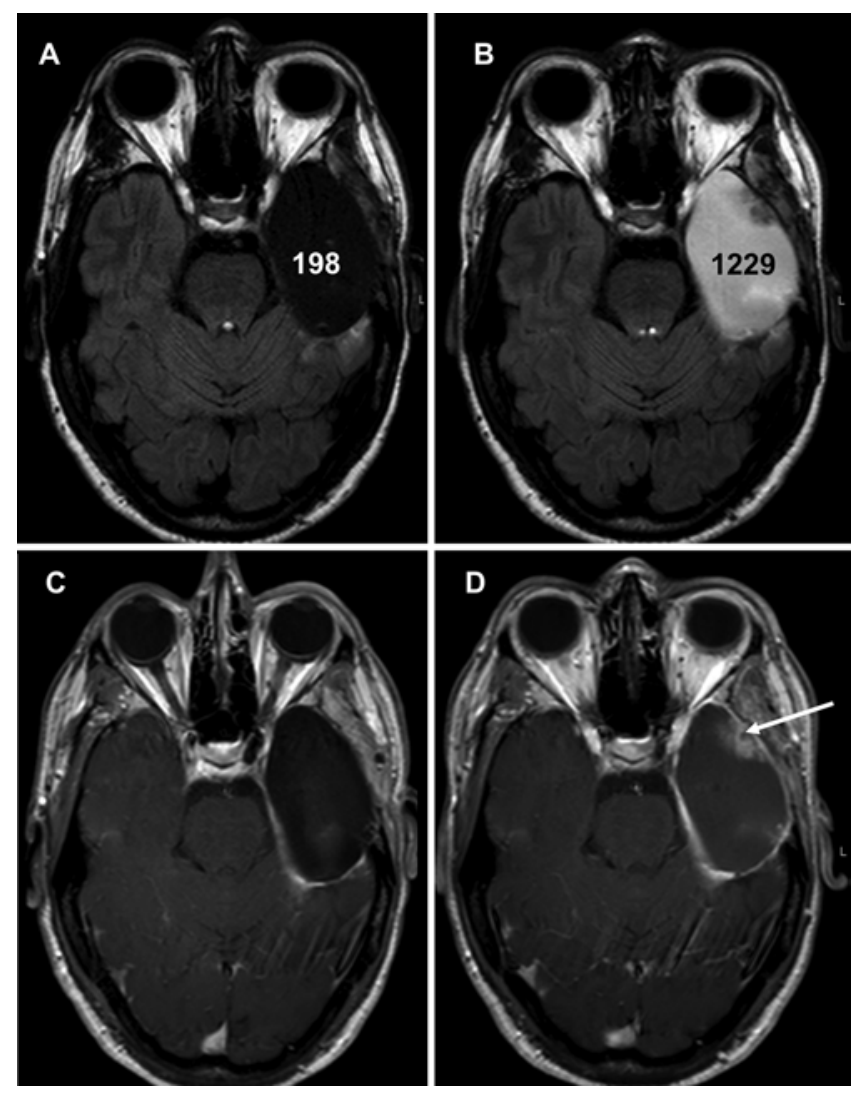

FIG. 5. Case of a patient with an anaplastic oligoastrocytoma (WHO Grade III) who had undergone previous surgery and radiotherapy. FLAIR image (A) and postcontrast T1-weighted image (C) obtained 11 months after surgery. FLAIR image obtained 4 months later (B), showing increase of signal intensity within the resection cavity. Corresponding postcontrast T1-weighted image (D) showing recurrent tumor (arrow) in the resection cavity.

of eloquent areas, as it is associated with improved prognosis. ${ }^{10,13,25,26}$ However, as the borders are poorly defined, it has to be assumed that there might be residual tumor cells despite complete resection of the contrast-enhancing tumor part. That supports the findings that FLAIR signal increase is also observed in patients who had undergone complete resection.

TABLE 3. Quantitative assessment of signal intensity of the fluid within the resection cavity

\begin{tabular}{lc}
\hline \multicolumn{1}{c}{ Variable } & Subgroup Analysis $(n=87)^{*}$ \\
\hline Signal intensity in resection cavity & $330.0(83.0-716.0)$ \\
Recurrent disease & $368.0(109.3-744.5)$ \\
No recurrent disease & $80.0(43.0-142.0)$ \\
\hline Specificity & $20.0 \%$ \\
Sensitivity & $62.2 \%$ \\
PPV for change & $92.7 \%$ \\
NPV for change & $3.2 \%$ \\
\hline
\end{tabular}

Nonnormally distributed data are shown as the median (IQR).

* Subgroup analysis of patients with WHO Grade III and IV gliomas, no connection of the resection cavity to CSF, and radiotherapy during the observation period.

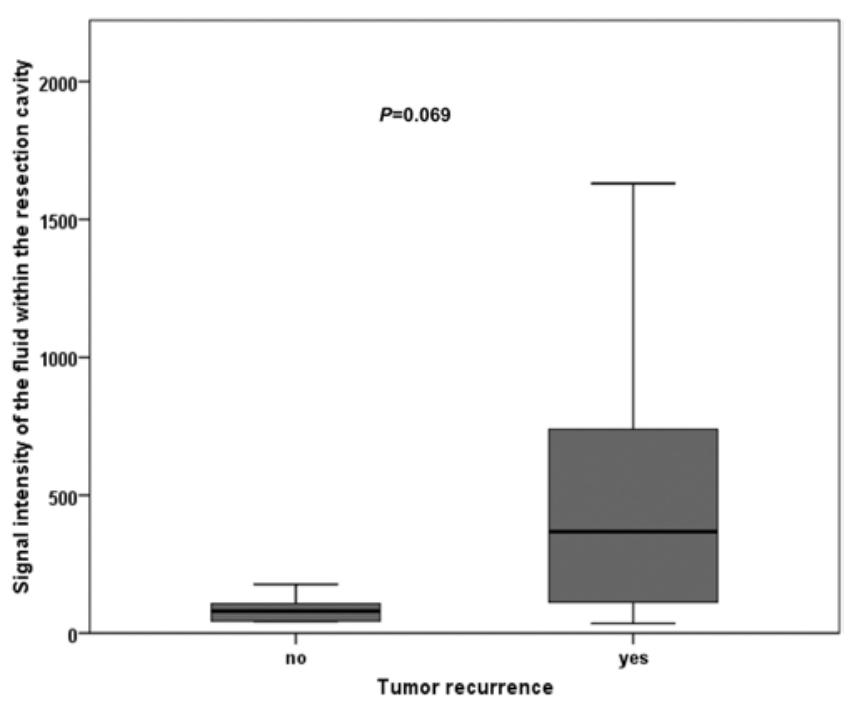

FIG. 6. Box plot for FLAIR signal intensity values of the fluid within the resection cavity in cases with and without recurrent disease.

Hemorrhage within the resection cavity resulting in signal intensity increase was discussed in the previous study, but it was not observed in the cases with recurrent disease. ${ }^{36}$ In our study we did not observe cases of bleeding within the resection cavity, which can cause an increase in FLAIR signal. Bleeding is described as a rare complication of intracranial glioma. ${ }^{2,15}$ Studies have shown that signal increase in FLAIR sequences is observed in bleeding and in cases of infarction due to vasogenic edema and a disrupted blood-brain barrier. ${ }^{12,21}$ Both bleeding and postoperative infarction are observed after glioma surgery, resulting in a FLAIR signal increase in the resection cavity. ${ }^{6-8}$ Therefore, the evaluation of FLAIR signal on the image obtained immediately postoperatively is not reliable and was excluded in this study. Only an increase in FLAIR signal at follow-up might predict tumor recurrence with high specificity. To avoid misinterpretation of bleeding, consideration of T1-weighted sequences without contrast agent is important. ${ }^{18}$

Infection within the resection cavity was observed in 2 cases, and these cases were excluded from analysis. It is known that an intracranial abscess exhibits an increase in FLAIR signal intensity due to a higher protein concentration. $^{20}$ Therefore-in addition to bleeding-infection could be another shortcoming of this method. In our study, none of the patients included in the study population with FLAIR signal intensity increase showed any signs of infection. However, in these rare cases, other MRI sequences such as T1-weighted images with and without contrast agent and diffusion-weighted imaging as well as clinical parameters could help determine the correct diagnosis. ${ }^{23}$

Quantitative analysis confirmed the results of the qualitative analysis but did not add a further diagnostic value. Therefore, qualitative analysis might be sufficient in the clinical routine. Both qualitative and quantitative analyses showed high interrater reliability, supporting practicability.

Sensitivity of this method is low in comparison with other methods that are used for detection of tumor recurrence, such as perfusion-weighted imaging and PET im- 
aging. ${ }^{4,27,29}$ However, one must consider that FLAIR sequences are included in the standard imaging protocol in glioma patients, and no additional MRI time or even radiation exposure is necessary for this method. Due to the high specificity of FLAIR signal increase for tumor recurrence/ tumor progression, observation of this sign should implicate adjustment of patients' surveillance and shortening of monitoring intervals. However, as this sign is infrequently seen, exclusion of tumor progression is not possible.

The main limitation of this study is its retrospective design. Gliomas are a heterogeneous group of tumors. Except for the analyzed subgroups of partially/completely resected and irradiated/nonirradiated gliomas, we did not assess other factors that influence tumor biology of gliomas, such as molecular markers or histopathological subtypes. These factors might have an impact on signal intensity in gliomas and should be addressed in further studies. Another limitation might arise due to the fact that quantitative and qualitative analysis of FLAIR signal intensity was performed on MR scanners with different sequence parameters. To reduce this bias, ratios of FLAIR signal intensity of the resection cavity to the CSF were calculated as described before in a previous study. ${ }^{36}$

\section{Conclusions}

An increase in FLAIR signal intensity of the fluid within the resection cavity of gliomas is a highly specific and early sign for tumor recurrence/tumor progression and can easily be used in the clinical routine. As this sign is mainly observed in patients with high-grade gliomas (WHO III and IV), radiotherapy, and without connection of the resection cavity to CSF, its use should be limited to this subgroup.

No differences were observed for gliomas with previous complete or incomplete tumor resection. Observation of FLAIR signal increase should dictate shortening of surveillance intervals to timely detect tumor progression or tumor recurrence. The possible pathomechanism is disturbance of CSF exchange due to an encapsulation of the resection cavity by tumor cells, which is supported by the findings that it mainly occurs in cases without connection of the resection cavity to CSF. Further studies need to be performed to confirm this hypothesis.

\section{Acknowledgments}

We thank Melanie Barz from the Department of Neurosurgery for recording of clinical data.

\section{References}

1. Arvold ND, Reardon DA: Treatment options and outcomes for glioblastoma in the elderly patient. Clin Interv Aging 9:357-367, 2014

2. Della Puppa A, Zustovich F, Gardiman M, Manara R, Cecchin D, Scienza R: Haemorrhagic presentation of low-grade glioma in adults. Acta Neurochir (Wien) 149:1151-1155, 2007

3. Duffau H, Taillandier L: New concepts in the management of diffuse low-grade glioma: Proposal of a multistage and individualized therapeutic approach. Neuro Oncol 17:332-342, 2015

4. Galldiks N, Dunkl V, Stoffels G, Hutterer M, Rapp M, Sabel
M, et al: Diagnosis of pseudoprogression in patients with glioblastoma using O-(2-[18 F]fluoroethyl)-L-tyrosine PET. Eur J Nucl Med Mol Imaging 42:685-695, 2015

5. Galldiks N, Stoffels G, Filss C, Rapp M, Blau T, Tscherpel $\mathrm{C}$, et al: The use of dynamic O-(2- ${ }^{18} \mathrm{~F}$-fluoroethyl)-L-tyrosine PET in the diagnosis of patients with progressive and recurrent glioma. Neuro Oncol 17:1293-1300, 2015

6. Gempt J, Förschler A, Buchmann N, Pape H, Ryang YM, Krieg SM, et al: Postoperative ischemic changes following resection of newly diagnosed and recurrent gliomas and their clinical relevance. J Neurosurg 118:801-808, 2013

7. Gempt J, Gerhardt J, Toth V, Hüttinger S, Ryang YM, Wostrack M, et al: Postoperative ischemic changes following brain metastasis resection as measured by diffusion-weighted magnetic resonance imaging. J Neurosurg 119:1395-1400, 2013

8. Gempt J, Krieg SM, Hüttinger S, Buchmann N, Ryang YM, Shiban E, et al: Postoperative ischemic changes after glioma resection identified by diffusion-weighted magnetic resonance imaging and their association with intraoperative motor evoked potentials. J Neurosurg 119:829-836, 2013

9. General Assembly of the World Medical Association: World Medical Association Declaration of Helsinki: ethical principles for medical research involving human subjects. J Am Coll Dent 81:14-18, 2014

10. Grabowski MM, Recinos PF, Nowacki AS, Schroeder JL, Angelov L, Barnett GH, et al: Residual tumor volume versus extent of resection: predictors of survival after surgery for glioblastoma. J Neurosurg 121:1115-1123, 2014

11. Ito-Yamashita T, Nakasu Y, Mitsuya K, Mizokami Y, Namba $\mathrm{H}$ : Detection of tumor progression by signal intensity increase on fluid-attenuated inversion recovery magnetic resonance images in the resection cavity of high-grade gliomas. Neurol Med Chir (Tokyo) 53:496-500, 2013

12. Jha R, Battey TW, Pham L, Lorenzano S, Furie KL, Sheth $\mathrm{KN}$, et al: Fluid-attenuated inversion recovery hyperintensity correlates with matrix metalloproteinase-9 level and hemorrhagic transformation in acute ischemic stroke. Stroke 45:1040-1045, 2014

13. Keles GE, Chang EF, Lamborn KR, Tihan T, Chang CJ, Chang SM, et al: Volumetric extent of resection and residual contrast enhancement on initial surgery as predictors of outcome in adult patients with hemispheric anaplastic astrocytoma. J Neurosurg 105:34-40, 2006

14. Le Rhun E, Taillibert S, Chamberlain MC: The future of high-grade glioma: Where we are and where are we going. Surg Neurol Int 6 (Suppl 1):S9-S44, 2015 (Erratum in Surg Neurol Int 6:37, 2015)

15. Licata B, Turazzi S: Bleeding cerebral neoplasms with symptomatic hematoma. J Neurosurg Sci 47:201-210, 2003

16. Louis DN, Ohgaki H, Wiestler OD, Cavenee WK, Burger PC, Jouvet A, et al: The 2007 WHO classification of tumours of the central nervous system. Acta Neuropathol 114:97-109, 2007

17. Lu S, Ahn D, Johnson G, Law M, Zagzag D, Grossman RI: Diffusion-tensor MR imaging of intracranial neoplasia and associated peritumoral edema: introduction of the tumor infiltration index. Radiology 232:221-228, 2004

18. Meyding-Lamadé U, Forsting M, Albert F, Kunze S, Sartor $\mathrm{K}$ : Accelerated methaemoglobin formation: potential pitfall in early postoperative MRI. Neuroradiology 35:178-180, 1993

19. Min ZG, Niu C, Rana N, Ji HM, Zhang M: Differentiation of pure vasogenic edema and tumor-infiltrated edema in patients with peritumoral edema by analyzing the relationship of axial and radial diffusivities on 3.0T MRI. Clin Neurol Neurosurg 115:1366-1370, 2013

20. Mishra AM, Reddy SJ, Husain M, Behari S, Husain N, Prasad KN, et al: Comparison of the magnetization transfer 
ratio and fluid-attenuated inversion recovery imaging signal intensity in differentiation of various cystic intracranial mass lesions and its correlation with biological parameters. J Magn Reson Imaging 24:52-56, 2006

21. Noguchi K, Ogawa T, Inugami A, Toyoshima H, Sugawara S, Hatazawa J, et al: Acute subarachnoid hemorrhage: MR imaging with fluid-attenuated inversion recovery pulse sequences. Radiology 196:773-777, 1995

22. Price SJ, Jena R, Burnet NG, Hutchinson PJ, Dean AF, Peña $A$, et al: Improved delineation of glioma margins and regions of infiltration with the use of diffusion tensor imaging: an image-guided biopsy study. AJNR Am J Neuroradiol 27:1969-1974, 2006

23. Reiche W, Schuchardt V, Hagen T, Il'yasov KA, Billmann P, Weber J: Differential diagnosis of intracranial ring enhancing cystic mass lesions-role of diffusion-weighted imaging (DWI) and diffusion-tensor imaging (DTI). Clin Neurol Neurosurg 112:218-225, 2010

24. Salcman M: Survival in glioblastoma: historical perspective. Neurosurgery 7:435-439, 1980

25. Stummer W, Pichlmeier U, Meinel T, Wiestler OD, Zanella F, Reulen HJ: Fluorescence-guided surgery with 5-aminolevulinic acid for resection of malignant glioma: a randomised controlled multicentre phase III trial. Lancet Oncol 7:392 401, 2006

26. Stummer W, Reulen HJ, Meinel T, Pichlmeier U, Schumacher W, Tonn JC, et al: Extent of resection and survival in glioblastoma multiforme: identification of and adjustment for bias. Neurosurgery 62:564-576, 2008

27. Suh CH, Kim HS, Choi YJ, Kim N, Kim SJ: Prediction of pseudoprogression in patients with glioblastomas using the initial and final area under the curves ratio derived from dynamic contrast-enhanced T1-weighted perfusion MR imaging. AJNR Am J Neuroradiol 34:2278-2286, 2013

28. Terakawa Y, Yordanova YN, Tate MC, Duffau H: Surgical management of multicentric diffuse low-grade gliomas: functional and oncological outcomes: clinical article. J Neurosurg 118:1169-1175, 2013

29. Thomas AA, Arevalo-Perez J, Kaley T, Lyo J, Peck KK, Shi W, et al: Dynamic contrast enhanced T1 MRI perfusion differentiates pseudoprogression from recurrent glioblastoma. J Neurooncol 125:183-190, 2015

30. Urbańska K, Sokołowska J, Szmidt M, Sysa P: Glioblastoma multiforme-an overview. Contemp Oncol (Pozn) 18:307312,2014

31. van den Bent MJ, Wefel JS, Schiff D, Taphoorn MJ, Jaeckle $\mathrm{K}$, Junck L, et al: Response assessment in neuro-oncology (a report of the RANO group): assessment of outcome in trials of diffuse low-grade gliomas. Lancet Oncol 12:583-593, 2011

32. Weller M, van den Bent M, Hopkins K, Tonn JC, Stupp R, Falini A, et al: EANO guideline for the diagnosis and treatment of anaplastic gliomas and glioblastoma. Lancet Oncol 15:e395-e403, 2014

33. Wen PY, Macdonald DR, Reardon DA, Cloughesy TF, Sorensen AG, Galanis E, et al: Updated response assessment criteria for high-grade gliomas: response assessment in neuro-oncology working group. J Clin Oncol 28:1963-1972, 2010

34. Wick W, Weller M: Classification and management of anaplastic gliomas. Curr Opin Neurol 22:650-656, 2009

35. Wick W, Wiestler B, Platten M: Treatment of anaplastic glioma. Cancer Treat Res 163:89-101, 2015

36. Winterstein M, Münter MW, Burkholder I, Essig M, Kauczor HU, Weber MA: Partially resected gliomas: diagnostic performance of fluid-attenuated inversion recovery MR imaging for detection of progression. Radiology 254:907-916, 2010

\section{Disclosures}

The authors report the following. Prof. Zimmer has served on scientific advisory boards for Philips and Bayer-Schering; serves as co-editor on the advisory board of Clinical Neuroradiology; has received speaker honoraria from Bayer-Schering and Philips; and has received research support and investigator fees for clinical studies from Biogen Idec, Quintiles, MSD Sharp \& Dome, Boehringer Ingelheim, Inventive Health Clinical UK Ltd., Advance Cor, Brainsgate, Pfizer, Bayer-Schering, Novartis, Roche, Servier, Penumbra, WCT GmbH, Syngis, SSS International Clinical Research, PPD Germany GmbH, Worldwide Clinical Trials Ltd., Phenox, Covidien, Actelion, Medivation, Medtronic, Harrison Clinical Research, Concentric, Pharmtrace, Reverse Medical Corp., Premier Research Germany Ltd., Surpass Medical Ltd., and GlaxoSmithKline. Drs. Bette, Huber, and Gempt and Prof. Meyer work as consultants for Brainlab (Brainlab AG, Feldkirchen).

\section{Author Contributions}

Conception and design: Bette, Gempt, Boeckh-Behrens . Acquisition of data: Bette, Huber, Delbridge. Analysis and interpretation of data: Bette, Gempt, Boeckh-Behrens. Drafting the article: Bette. Critically revising the article: Gempt, Huber, Delbridge, Meyer, Zimmer, Kirschke, Boeckh-Behrens. Reviewed submitted version of manuscript: Gempt, Huber, Delbridge, Meyer, Zimmer, Kirschke, Boeckh-Behrens. Approved the final version of the manuscript on behalf of all authors: Bette. Statistical analysis: Bette, Gempt, Boeckh-Behrens. Study supervision: Gempt, Meyer, Zimmer, Kirschke, Boeckh-Behrens.

\section{Online-Only Content}

Supplemental material is available with the online version of the article.

$$
\text { Supplemental Table 1. https://thejns.org/doi/suppl/ }
$$
10.3171/2016.8.JNS16752.

\section{Correspondence}

Stefanie Bette, Klinikum rechts der Isar, Technische Universität München, Ismaningerstr. 22, Munich 81675, Germany. email: stefanie.bette@tum.de. 